\title{
Avaliação da percepção e autocuidado em Saúde Bucal na Atenção Básica na perspectiva do envelhecimento
}

\section{Evaluation of perception and self-care in oral health in Basic Care in the aging perspective}

\section{Evaluación de la percepción y autocuidado en Salud Bucal en laAtención Básica en la perspectiva del envejecimiento}

Ermano Batista Costa ${ }^{1}$,

RESUMO: Os adultos foram ao longo dos anos mitigados pela odontologia curativo-mutiladora, sem receberem, via de regra, tratamentos preventivos. Escassos são os estudos em saúde bucal com intuito de conhecer o que os adultos pensam e quais os cuidados praticam na vida cotidiana. Neste sentido, esta pesquisa objetivou avaliar a percepção e o autocuidado em saúde bucal de adultos na perspectiva do envelhecimento. Com a abordagem quantiqualitativa,utilizou-se um questionário para entrevista semiestruturada, apresentando os resultados através de estatística descritiva e exposição dos melhores discursos. Foram entrevistados 50 adultos, entre 25 a 59 anos, usuários de uma Unidade de Básica de Saúde (UBS)em Nova Russas-CE, Brasil. Predominouno grupo de entrevistados aqueles do sexo feminino (78\%), pardos (80\%), com ensino fundamental incompleto (42\%), beneficiários do programa Bolsa Família (62\%), ocupação do lar (42\%) e idade média de 40,66 anos. A maioria dos usuários não acredita que terá dentes naturais quando idosos (70\%) e 
já percebem sinais de envelhecimento (74\%). Poucos conhecem o que é cálculo dentário (8\%),e já perderam vários elementos dentários (6,67 dentes/pessoa). Os usuários apresentaram valores arraigados como escovar os dentes ao acordar (88\%) ao invés de depois do café,e alguns procuram atendimento odontológico quando aparecem queixas: $28 \%$ ao sentir dor de dente e $34 \%$ quando consideram precisar. Em suma, os usuários mostraram uma relação de codependência ao sistema de saúde pública vigente, havendo a necessidade desse ser efetivamente integral e universal,ao passo que os usuários busquem corresponsabilizar-se pela sua saúde oral, cuidando-se autonomamente.

Palavras-chave: Saúde Bucal, Envelhecimento, Autopercepção, Autoavaliação.

\section{INTRODUÇÃO}

No Brasil, em termos de saúde pública, há um quadro precário de saúde bucal na faixa etária adulta e idosa, resultado possivelmente da ausência de programas governamentais específicos, que busquem enfatizar a autoproteção e autopercepção através da educação em saúde ${ }^{1,2}$. Ainda se constata ações educativas geralmente voltadas a certos grupos como as crianças e adolescentes, ficando à margem os adultos, importantes agentes multiplicadores de informações no núcleo familiar ${ }^{3}$.

A história pregressada saúde bucal retratam condições de indivíduos que vivenciaram o modelo tradicional curativista, caracterizado por serviços escassos e de acesso restrito, o qual expôs os indivíduos a resolução pontual da dor pela opção fatídica da remoção dentária ${ }^{1,4,5}$. Logo, a maioria da população idosa brasileira apresenta elevado número de dentes perdidose consequente necessidade de uso de prótese 6 .

O envelhecimento é vivido de maneira diferente de indivíduo para indivíduo, por gerações e pelas sociedades ${ }^{6}$. Esse processo é entendido como biológico, socioeconômico e psicossocial, além disso, é contínuo, não se iniciando em nenhuma idade ou momento particular ${ }^{7}$. A boca desdentada, então, no processo de envelhecimento, adquire significados que são interdependentes de fatores, desde os históricos até os fisiológicos ${ }^{4,8}$.

Considerando-se que o comportamento dos indivíduos é condicionado por suas percepções e pela importância dada a elas, a avaliação da autopercepção das condições de saúde bucal representa um ganho substancial para o planejamento dos serviços de saúde ${ }^{9}$. Observa-se que, parte das pessoas não procura atendimento odontológico pela não percepção de suas necessidades, quando se atentam a isso, usam critérios diferentes dos empregados pelo profissional ${ }^{10}$.

$\mathrm{Na}$ idade adulta, doenças como lesões de tecidos moles e duros, incluindo o câncer bucal, problema periodontal e xerostomia são recorrentes ${ }^{11}$.A exodontia é o pior resultado da doença cárie entre os adultos aos 40 anos, agravada a situação pela cárie de raiz ${ }^{12}$. Frente à desanimadora realidade da condição de saúde bucal dos adultos e idosos brasileiros, estudos em saúde pública suscitam o levantamento de novas hipóteses, para que a atenção em saúde bucal mude consubstancialmente ${ }^{13}$. 
Evidencia-se na prática clínica que adultos, e idosos principalmente, aparecem no consultório odontológico da Unidade Básica de Saúde (UBS), por vezes, apenas para realizar exodontias. Eles têm a cultura arraigada de remover dentes, e é difícil convencê-los do contrário. Caso não seja realizado o procedimento, o usuário geralmente procura um profissional que o faça. Se convencido a permanecer com os dentes, o indivíduo não cuida como deveria, e perdê-los é provável. Assim, avaliou-se a percepção e o autocuidado em saúde bucal de usuários adultos de uma UBS situada em município do interior cearense.

\section{METODOLOGIA}

O estudo é transversal, exploratório e descritivo. Optou-se pela abordagem quantitativa e qualitativa. Ao combiná-las, procurou-se evitar as fragilidades próprias de cada método, aumentado a validade de seus constructos ${ }^{14}$. Realizou-se na UBS do distrito de Espacinha, localizada a 10 $\mathrm{km}$ da área urbana do seu município Nova Russas - Ceará, Brasil, a qual atende a uma área de abrangência em torno de 554 famílias.

A amostra não probabilística foi composta por 50 adultos, selecionada aleatoriamente, conforme os indivíduos procuravam a unidade de saúde para realizar a Primeira Consulta Odontológica Programática, que consiste na avaliação das condições gerais de saúde e realização de exame clínico odontológico com finalidade de diagnóstico e, necessariamente, elaboração de plano preventivoterapêutico. Esse tipo de consulta é registrado novamente para a mesma pessoa 12 meses após a conclusão do plano preventivo-terapêutico ou caso o paciente abandone o tratamento seis meses após a última consulta ${ }^{15}$.

Esta pesquisa é caracterizada como estudo de caso, o qual é circunscrito a poucas unidades de análise, entendidas estas como: pessoas, famílias, produtos, instituições, comunidades, regiões e países. Como se aplica a poucas unidades de análise, a riqueza não está na extensão, mas na profundidade deste tipo de pesquisa ${ }^{16}$.

Para delimitar o número de entrevistas, considerou-se o critério de saturação teórica das informações, frequentemente empregado nos relatórios de investigações qualitativas para estabelecer o término da coleta dos dados, interrompendo a captação de novos componentes ${ }^{17}$.

Os dados foram coletados entre janeiro e fevereiro de 2015, através de entrevistas semiestruturadas, realizadas privativamente por único pesquisador treinado, e gravadas em aparelho portátil, seguindo um roteiro de questões abertas e fechadas que geraram um formulário com as seguintes variáveis: idade, sexo, cor, escolaridade, ocupação, renda individual, percepção sobre envelhecimento dentário, autocuidado bucal e perda dentária. Esse instrumento foi baseado numa versão simplificada do Oral HealthImpact Profile, o OHIP-14, preconizadopor Slade (1997) ${ }^{18}$,e no Oral Health Questionare for Adults da World Health Organization ${ }^{19}$.

Um projeto piloto foi conduzido anteriormente à coleta de dados com 5 adultos $(10 \%$ da amostra ISSN 1982-8829 Tempus, actas de saúde colet, Brasília, 13(3), 93-105, set, 2019. Epub Jul/2020 
total), nele o método teste/reteste foi empregado para verificar a confiabilidade e o desempenho do instrumento de coleta de dados ${ }^{20}$.

Parte das respostas obtidas foi tabulada pelo Software da Microsoft, o Excel 2010, sendo osdemais dados analisados com base na teoria do Discurso do Sujeito Coletivo (DSC), que se caracteriza como uma proposta de organização e de tabulação de dados qualitativos, extraindo de cada um dos depoimentos as ideias centrais, as ancoragens e as expressões-chaves, emitidas na primeira pessoa do singular. Assim, o DSC consiste na unificação e no agrupamento de vários sujeitos emissores de discurso, permitindo que se coletivizem seus discursos, expressando diretamente a opinião de um dado sujeito social no cerne natural do funcionamento das representações sociais ${ }^{21-23}$. Para manter a privacidade dos usuários, seus discursos foram expostos, recebendo a letra $U$, acompanhada de números na ordem em que ocorreram as entrevistas.

A média aritmética foi usada na estatística descritiva dos dados numéricos, seguida do valor mínimo e máximo, bem como calculado o Desvio Padrão (DP), o qual demonstra a regularidade referente a um conjunto de dados de modo a apontar o grau de oscilação destes em comparação a média dos valores do conjunto. A simplicidade da análise utilizada nesta pesquisa objetivou favorecer o entendimento dos cirurgiões-dentistas que atuam nas UBSs, por crer-se na limitada busca e acesso destes profissionais a multiplicidade e a complexidade de metodologias estatísticas encontradas no meio científico.

Este estudo foi conduzido de acordo com os preceitos determinados pela Resolução 466/12 para estudo com seres humanos, do Conselho Nacional de Saúde do Ministério da Saúde, sob o Parecer: 660.902 /CAAE: 24721914.8.0000.5054, consubstanciado pelo Comitê de Ética em Pesquisa da Universidade Federal do Ceará.

\section{RESULTADOS E DISCUSSÃO}

A média de idade do grupo estudado foi de 40,66 anos (Mín. 26 e Máx. 59) e o DP=8,012.A faixa etária de adultos parece ser a mais adequada para estudar os problemas bucais, devido ao seu maior tempo com dentição permanente na cavidade oral, além de possibilitar a verificação do impacto de uso dos serviços odontológicos ${ }^{5}$. Sendo os dados socioeconômicos apresentados na tabela 1.

Tabela 1: Distribuição absoluta e percentual dos dados socioeconômicos dos usuários entrevistados, ESF Espacinha, Nova Russas-CE.

\begin{tabular}{llll}
\hline DADOS SOCIOECONÔMICOS & n & $\%$
\end{tabular}

\begin{tabular}{llll}
\hline \multirow{2}{*}{ SEXO } & Feminino & 39 & 78 \\
& Masculino & 11 & 22 \\
\cline { 2 - 4 }
\end{tabular}




\begin{tabular}{|c|c|c|c|}
\hline \multirow{2}{*}{ COR } & Brancos & 10 & 20 \\
\hline & Não brancos (parda) & 40 & 80 \\
\hline \multirow{2}{*}{ RENDA } & Bolsa Família & 31 & 62 \\
\hline & 1 Salário Mínimo & 19 & 38 \\
\hline \multirow{5}{*}{ ESCOLARIDADE } & Fundamental incompleto & 26 & 52 \\
\hline & Fundamental completo & 4 & 8 \\
\hline & Médio incompleto & 4 & 8 \\
\hline & Médio completo & 14 & 28 \\
\hline & Superior & 2 & 4 \\
\hline \multirow{7}{*}{ OCUPAÇÃO } & Comerciante & 5 & 10 \\
\hline & Crocheteira* & 8 & 16 \\
\hline & Do lar & 21 & 42 \\
\hline & Agricultor (a) & 7 & 14 \\
\hline & Aposentado (a) & 3 & 6 \\
\hline & Servidor (a) público & 6 & 12 \\
\hline & Total & 50 & 100 \\
\hline
\end{tabular}

Fonte: autoria própria.

*O município de Nova Russas é considerado a capital mundial do crochê, que é a arte de fazer e decorar roupas de vestir, de cama, mesa e banho pelas mãos de mulheres habilidosas - as crocheteiras.

\section{Dados socioeconômicos}

O sexo predominante no estudo foi o feminino com $78 \%$ contra $22 \%$ de masculino. Estudos em saúde coletiva ${ }^{24-26}$, que se utilizaram de usuários em atendimento, confirmam a predominância de entrevistados do sexo feminino sobre o masculino, explicada pela disciplina das mulheres no cuidado à saúde e por terem mais tempo disponível quando cuidam apenas do lar. Sobre a cor, 80\% dos usuários declararam-se não brancos, utilizando os seguintes termos para descrição: moreno (a), queimado (a), da cor de canela, escuro (a), sendo tais termos não apresentados, e categorizados, portanto, como cor parda.

A maioria dos usuários era de beneficiário do programa federal Bolsa Família $62 \%$, enquanto $38 \%$ disseram ter renda individual em torno de 1 salário-mínimo.Nos entrevistados predominaram ainda a escolaridade fundamental incompleto com $52 \%$ e a ocupação do lar com $42 \%$, corroborando com a pesquisa de Fonseca, Nehmy e Mota (2015) ${ }^{27}$. Sabe-se que um maior nível de escolaridade e renda favorece mais busca por informações de saúde e uma autoavaliação positiva da saúde ${ }^{20}$. No entanto, Fonseca, Nehmy e Mota $(2015)^{27}$ notaram que quando a escolaridade destoa do conjunto dos participantes da pesquisa, não se observamdiferenças na percepção dos entrevistados, o que pode ser atribuído à semelhança da posição social em relação à renda e a consequente dependência dos serviços odontológicos do SUS. 
A tabela 2 expõe a percepção e o autocuidado oral dos usuários na perspectiva do envelhecimento.

Tabela 2: Distribuição percentual e absoluta dos usuários quanto a sua percepção e autocuidado oral na perspectiva do envelhecimento, ESF Espacinha, Nova Russas-CE.

\begin{tabular}{lcccccc}
\hline \multirow{2}{*}{ IDEIAS CENTRAIS } & \multicolumn{2}{c}{ Sim } & \multicolumn{2}{c}{ Não } & & Em dúvida \\
\cline { 2 - 7 } & n & \% & n & \% & n & \% \\
\hline Percebe dentes envelhecendo & 37 & 74 & 13 & 26 & 0 & 0 \\
Percebe dentes escurecendo & 41 & 82 & 6 & 12 & 3 & 6 \\
Percebe dentes quebrando & 27 & 54 & 23 & 46 & 0 & 0 \\
Percebe dentes desgastando & 32 & 64 & 17 & 34 & 1 & 2 \\
Percebe dentes moles & 5 & 10 & 45 & 90 & 0 & 0 \\
Percebe gengiva retraindo & 12 & 24 & 32 & 64 & 6 & 12 \\
Tem conhecimento sobre tártaro & 4 & 8 & 46 & 92 & 0 & 0 \\
Pós-informado conhece o tártaro & 28 & 56 & 22 & 44 & 0 & 0 \\
Escovação dentária antes de & 47 & 94 & 0 & 0 & 3 & 6 \\
dormir & & & & & & \\
Usa fio dental regularmente & 29 & 58 & 17 & 34 & 4 & 8 \\
Usa limpador de língua & 8 & 16 & 42 & 84 & 0 & 0 \\
Usa antisséptico bucal & 15 & 30 & 35 & 70 & 0 & 0 \\
Já fez tratamento de canal & 14 & 28 & 36 & 72 & 0 & 0 \\
Acredita se terá dentes quando & 11 & 22 & 35 & 70 & 4 & 8 \\
idoso & & & & & & \\
Acredita no SUS do futuro & 29 & 58 & 16 & 32 & 5 & 10 \\
\hline
\end{tabular}

Fonte: autoria própria.

\section{Autopercepção}

Dos usuários entrevistados, $26 \%$ não percebiam sinais de envelhecimento dentário, enquanto $74 \%$ percebiam: escurecimento $82 \%$, dentes quebrando $54 \%$ e desgastando $64 \%$. Assemelhamse a estes resultados os de Fonseca, Nehmy e Mota (2015) ${ }^{27}$, em que parte das pessoas descreve seus dentes de forma negativa como 'ruins, tortos e muito frágeis, que foram se estragando ou se quebrando com muita facilidade'. Com o avançar da idade, 39,5\% das pessoas afirmam possuir problemas com os dentes e $36,8 \%$ com as gengivas ${ }^{2}$.

A percepção sobre os problemas periodontias pareceu confusa no presente estudo, visto que apenas $10 \%$ sentiam dentes moles e $24 \%$ observavam gengiva retraindo. Faz-se necessário a realização de estudos avaliativos clínicos para diagnosticar in locu os problemas de ordem periodontal que por ventura os usuários da atenção primária apresentem e não percebam. A validade da concordância entre avaliação normativa (clínica) e autopercebida sobre essa doença tem mostrado resultados 
inconsistentes.Logo, a percepção do público deve ser mais condizente com a avaliação normativa dos profissionais, e estes devem estar mais envolvidos com as necessidades de saúde bucal percebidas da população, em que a prevalência da doença periodontal em adultos brasileiros é de $8,9 \%{ }^{28}$.

Poucos usuários afirmaram ter conhecimento sobre o que éo cálculo dentário $8 \%$, denominado na pesquisa como tártaro para um melhor entendimento na entrevista. Após explicação do conceito de tártaro, 56\% afirmaram que tinham ou já apresentaram tal condição. No grupo etário de 35 a 44 anos, o Projeto Saúde Bucal (SB) 2010 observou que a presença de cálculo foi a condição mais expressiva, presente em $28,6 \%$ dos adultos examinados. A presença de cálculo dentário aumenta com a idade, atingindo a maior prevalência entre adultos, aproximadamente $64 \%$, declinando nos $\operatorname{idosos}^{29}$.

\section{Autocuidado}

Quando questionado se os usuários escovavam os dentes antes ou depois do café da manhã, 88\% dos usuários falaram que escovavam antes, e apenas $6 \%$ disseram que escovavam depois. Nesta mesma porcentagem, afirmaram fazer a higienização oral antes e depois de tomar o café (dados não apresentados). Já escovação dentária antes de dormir foi relatada pela maioria dos entrevistados (94\%) - Tabela 1. A média do número de escovações dentárias por dia resultou em 2,6 vezes (Mín. 2 e Máx. 3), $\mathrm{DP}=0,495$.

Constatou-se que todos os usuários entrevistados no presente estudo (100\%) utilizam creme dental como agente saponificante na escovação dentária (dados não apresentados). Sequencialmente, nenhum dos pesquisados $(0 \%)$ relatou usar terapias alternativas para higiene oral, além de fio dental $58 \%$ e/ou limpador de língua 16\% e/ou antisséptico bucal 30\%. O uso de escova e de creme dental não apresenta relação com o impacto das condições bucais na qualidade de vida, sendo maior o impacto entre os indivíduos que relatam nunca usar fio dental e menor entre os que afirmam usar sempre $^{10}$.

Indistintamente, os usuários que realizaram tratamento endodôntico (28\%) e aqueles que ainda não o fizeram (72\%), afirmaram que fariam este tipo de tratamento na tentativa de não perder seu elemento dentário, usando expressões do tipo: Faria para não perder o dente (U1); Fazia outro canal se precisasse (U2). Tais discursos parecem controversos, pois na prática eles resistem geralmente a esse tratamento, pelo mito da dor e pela crença fatídica da perda do elemento dentário. Além de mais, o sistema público de saúde bucal, através da atenção secundária dos CEOs, oferece poucas vagas para a realização da endodontia ${ }^{26}$.

\section{Confiabilidade}

Certas respostas foram mencionadas pelos usuários ao se indagar porque não acreditam que terão os dentes naturais quando idosos (70\%): Não porque já tão doendo os meus dentes (U10); 
Porque ficam estragando, aí tenho que extrair pra colocar outros (U9). Com o avançar da idade, acredita-se que devido à despreocupação com o tempo, os indivíduos se tornam mais tolerantes com os problemas surgidos na cavidade bucal ${ }^{6}$.Nos serviços públicos municipais de saúde, 64,7\% dos usuários acreditam que os dentes podem durar toda a vida, desde que se tomem atitudes como cuidar, tratar e ir ao dentista ${ }^{30}$.

Sobre o nível de confiabilidade no sistema público de saúde bucal, se o mesmo, através do SUS, é capaz de oferecer dentes saudáveis até a vida idosa, $58 \%$ acreditam que SIM, e utilizaram certas expressões como: A tendência é melhorar (U32); A medicina só evolui (U41). Alguns dos usuários que não acreditam no SUS do futuro (32\%), falaram: Se agora não presta, imagine depois (U1); Duvido, mas queria que sim (U28). É certo que todos os serviços odontológicos não estão disponíveis no SUS, e, portanto, nem sequer constam como opção, ficando a via privada como destino apenas para os indivíduos que apresentam condições socioeconômicas melhores ${ }^{27}$.

Ao perguntar os usuários em que ocasiões ou qual frequência procuravam atendimento odontológico, os resultados foram conforme a Tabela 3.

Tabela 3: Distribuição absoluta e percentual das ocasiões e frequência em que os usuários da ESF Espacinha procuram atendimento odontológico, Nova Russas-CE, Brasil.

\begin{tabular}{llllll}
\hline $\begin{array}{l}\text { OCASIÕES DE } \\
\text { ATENDIMENTO }\end{array}$ & $\mathbf{n}$ & $\mathbf{\%}$ & $\begin{array}{l}\text { FREQUÊNCIA DE } \\
\text { ATENDIMENTO }\end{array}$ & $\mathbf{n}$ & $\mathbf{\%}$ \\
\hline Realizar "limpeza" & 9 & 18 & 2 vezes por ano & 10 & 20 \\
Quando precisa & 17 & 34 & 1 vez a cada 2 ano & 2 & 4 \\
Dor de dente & 14 & 28 & 1 vez por ano & 15 & 30 \\
Precisa e sente dor & 5 & 10 & Apenas quando precisa & 17 & 34 \\
Para extração & 5 & 10 & Depende da necessidade & 2 & 4 \\
Não soube informar & 0 & 0 & Não soube informar & 4 & 8 \\
\hline Total & 50 & 100 & Total & 50 & 100 \\
\hline
\end{tabular}

Fonte: autoria própria.

\section{Ocasiões e frequência de atendimento}

No presente estudo, mencionou-se procurar atendimento odontológico após episódios de dor de dente em $28 \%$ dos casos, no entanto, nem sempre o serviço de saúde pública possui disponibilidade de material, instrumental, profissional e de vagas para atendimento ${ }^{31}$. Paralelamente, certos usuários demonstraram responsabilidade forçada com sua própria saúde bucal, ao mencionar que procura atendimento apenas quando precisa 34\%. É consensual, entre os cirurgiões-dentistas, a procura por atendimento odontológico público ou privado de 6 em 6 meses e/ou quando se perceber algum problema bucal.

O SB Brasil 2010 observou que o serviço público foi marcadamente o mais utilizado em todas as regiões na população geral, assim como a opção de ir ao dentista para prevenção ou tratamento. 
Destoando do presente estudo, em que $10 \%$ dos adultos buscam normalmente o atendimento odontológico apenas pra realizar extração dentária, esse mesmo estudo de âmbito nacional observou que na Região Norte, uma maior proporção de idosos (46,2\%) também procura o serviço apenas para extrair ${ }^{29}$. É certo que a odontalgia pode atingir todos os indivíduos, desde que possuam elementos dentários sem os devidos cuidados (Costa et.al, 2009).

\section{Perda dentária}

A média da perda de dentes perdidos relatados pelos usuários foi de 6,67 por pessoa (Mín. 0 e Máx. 22), $\mathrm{DP}=5,428)$. $\mathrm{O}$ adulto brasileiro de renda e escolaridade baixa acumula grande estoque de doenças bucais, o que se reflete no alto índice de perda dos dentes ${ }^{27}$. Perante os levantamentos epidemiológicos em saúde bucal nacional anteriores, o SB Brasil 2010 detectou constância no grau de perdas dentárias dos idosos (em torno de 27 dentes perdidos), o que em linhas gerais pode significar um menor ataque de cárie na população adulta de 35 a 44 anos, revelando também um maior acesso aos serviços odontológicos para realizar restaurações dentárias. Esta é uma importantíssima inversão de tendência registrada no País: os procedimentos mutiladores, representados pelas extrações dentárias, cedem espaço aos tratamentos restauradores ${ }^{29}$.

Quando foi questionado aos usuários que perderam dentes o porquê isso aconteceu, as respostas variaram: Não tinha orientação dos profissionais de saúde (U5); Deixei estragar, era uma criança(U14). A percepção da condição bucal e a importância dada a ela é que condicionam o comportamento do indivíduo. Enquanto o cirurgião dentista avalia a condição com base na ausência ou presença de doença, o paciente dá mais importância aos sintomas e problemas funcionais e sociais que são ocasionados pela presença de doenças².

E quando se indagou a quem ou a que os usuários culpariam a sua perda de dentes, os mesmos responderam: Porque não tinha profissional, naquele tempo era dificil (U5); A mim mesmo (U13). A leitura dos relatos obtidos por Fonseca, Nehmy e Mota $(2015)^{27}$ mostra que para parcela das pessoas entrevistadas, os serviços de saúde bucal não fizeram parte de sua vida passada, e, portanto, seus problemas dentários não foram percebidos como uma necessidade. Sendo evidente uma experiência regressa de pobreza e de familiares que influenciaram nas suas expectativas presente e futura em relação ao estado de seus dentes.

Quanto à substituição dos dentes perdidos, os usuários demostraram majoritariamente a perspectiva de colocar prótese dentária comumao invés de implantes dentários, justificando que: Nunca ouviu falar de implante de dente (U9); Não tenho condição financeira (U24); Tenho medo da cirurgia e rejeição (U7). Não corroborou com essas falas o estudo de Bulgarelli e Manço (2008) ${ }^{6}$, em que os sujeitos evocaram a vontade de realizar implantes dentários $(60 \%)$ e reabilitações fixas (40\%). Com isso, dirime-se que à luz do problema, os indivíduos devem tentar reverter estados como medo, falta de dinheiro e de conhecimento, passando a ter anseios de melhorar a qualidade de sua saúde bucal e consequentemente de vida.

ISSN 1982-8829 Tempus, actas de saúde colet, Brasília, 13(3), 93-105, set, 2019. Epub Ju1/2020 


\section{Considerações gerais}

Há uma crença de que usuários portadores de prótese total não necessitam de acompanhamento odontológico, bem como existe uma visão fatalista de que o idoso perde os dentes com a idade. Estes estigmas sobre a pessoa idosa devem ser combatidos por meio de práticas de prevenção, aumento da cobertura dos serviços para população mais jovem, melhoria dos hábitos de autocuidado e compreensão sobre necessidade de cuidados em saúde bucal ${ }^{1,30}$.

É preciso buscar uma Odontologia a qual não valorize somente a utilização de recursos clínicos para o diagnóstico das condições de saúde bucal, mas sim, que leve em consideração a forma como a população percebe sua condição de saúde bucal. Mesmo assim, a percepção da saúde bucal parece ter pouca influência nas condições clínicas ${ }^{2}$, mostrando ser necessário desenvolver ações preventivas e educativas para a população.

Fatores socioeconômicos e de desenvolvimento da autonomia dos indivíduos devem ser considerados nas estratégias de promoção da saúde ${ }^{5}$.A mobilização da comunidade e a participação de cada um como ator, e não mais como expectante, nos programas educativos, poderá favorecer o estabelecimento de práticas de autocuidado e aperfeiçoar a atenção à saúde bucal. Logo, a saúde é um dos principais elementos da qualidade de vida do indivíduo, influenciada diretamente pelo estilo de vida dos indivíduos ${ }^{32}$. Desta forma, não basta apenas transmitir conceitos e ensinar técnicas, é preciso demonstrar a contribuição positiva das mudanças na dieta alimentar e nos hábitos de higiene bucal para a melhoria das condições de vida da população ${ }^{3}$.

\section{CONCLUSÕES}

É importante a validação de instrumentos de pesquisa com a temática percepção e autocuidado em saúde bucal em adultos, dada à escassez na literatura científica, para que se possam obter ferramentas relevantes à construção de capacidades locais, instrumentalizando profissionais e gestores da saúde para tomada de decisão nos diferentes territórios de atuação da ESF.

É certo que a estrutura dentária vai se modificando ao longo dos anos e o usuário percebe isso como envelhecimento dentário. Há a necessidade de cuidados preventivos e quando não evitados, os curativos, em todas as faixas etárias para que o processo natural de envelhecimento não traga consequências danosas à saúde bucal do indivíduo.

Evidenciou-se que muitas pessoas adultas não acreditam que podem ter dentes saudáveis na terceira idade, embora até confiem que o SUS vai melhorar quando forem idosos. Ou seja, mostraram-se SUS dependentes, ao invés de darem credibilidade aos cuidados dentários individuais.

Neste contexto, percebeu-se o autocuidado em adultos, mas de forma tênue, pois as ações preventivas foram relatadas por vezes de forma ineficiente. Isso reforça a ideia de que os programas de promoção e prevenção em saúde bucal precisam ser intensificados,e assimilados pelos indivíduos 
enquanto adultospara que tenham dentes saudáveis na velhice.

\section{REFERÊNCIAS}

1. Brasil. Ministério da Saúde. Secretaria de Atenção à Saúde. Departamento de Atenção Básica. Saúde bucal: caderno de atenção básica. Brasília: Ministério da Saúde; 2006.

2. Bortoli D, Locatelli FA, Fadel CB, Baldani MH. Associação entre percepção de saúde bucal e indicadores clínicos e subjetivos: estudo em adultos de um grupo de educação continuada da terceira idade. Publ. Uepg Biol. Health Sci. 2003; 9 (3/4): 55-65.

3. Ferreira RI, Morano M Jr, Meneghim MC, Pereira AC. Dental health education for adult patients: report of an experience. Rev Odontol UNESP. 2004; 33(3): 149-56.

4. Piuvezam G, Ferreira AAA, Soares SCM, Souza DLB, Alves MSCF. As perdas dentárias sob a ótica do idoso. Odontologia Clín. Científ. 2006; 5(4): 299-306.

5. Silva-Junior MF, Fonseca EP, Batista MJ, Sousa MLR. Distribuição espacial das perdas dentárias em uma população de adultos. Rev Gaúch Odontol. 2017; 65(2): 115-120.

6. Bulgarelli AF, Manço ARX. Idosos vivendo na comunidade e a satisfação com a própria saúde bucal. Ciênc. Saúde Coletiva. 2008; 13(4): 1165-1174.

7. Cormack E. A saúde oral do idoso. 2002. Disponível em: www.odontologia.com.br/artigos/ geriatria.html. Acesso em: [15/01/2015].

8. Martins ES. A saúde bucal do adulto: uma proposição de plano de ação na atenção à saúde bucal do adulto no município de Augusto de Lima/MG. [Trabalho de Conclusão de Curso]. Belo Horizonte: Faculdade de Medicina da UFMG; 2010.

9. Haikal DS, Paula AMB, Martins AMEBL, Moreira AN, Ferreira EF. Autopercepção da saúde bucal e impacto na qualidade de vida do idoso: uma abordagem quanti-qualitativa. Ciênc. Saúde Coletiva. 2011; 16: 3317-29.

10. Coelho MP, Cordeiro MCP, Corrêa FF, Carvalho CM, Araújo VE. Avaliação do impacto das condições bucais na qualidade de vida medido pelo instrumento OHIP-14. UFES Rev Odontol. 2008; 10(3): 4-9.

11. Minas Gerais. Secretaria de Estado de Saúde. Atenção à saúde bucal. Belo Horizonte: SAS/MG; 2006.

12. Palmier AC, Ferreira EF, Mattos F, Vasconcelos M. Módulo saúde bucal no contexto da atenção básica á saúde - saúde do adulto; Belo Horizonte: Nescon UFMG e Coopmed; 2008. 
13. Nadanovsky P. O aumento da produção científica odontológica brasileira na saúde pública. Cad. Saúde Pública. 2006; 22(5): 1-2.

14. Kirschbaum C. Decisões entre pesquisas quali e quanti sob a perspectiva de mecanismos causais. RBCS. 2013; 28 (82): 181-91.

15. Brasil. Ministério da Saúde. Secretaria de Atenção à Saúde. e-SUS Atenção Básica: Manual do sistema com Coleta de Dados Simplificada. Brasília: Ministério da Saúde; 2016.

16. Tobar F, Yalour MR. Como fazer teses em saúde pública: conselhos e ideias para formular projetos e redigir teses e informes de pesquisa. Rio de Janeiro: Editora FIOCRUZ; 2003.

17. Fontanella BJB, Ricas J, Turato ER. Amostragem por saturação em pesquisas qualitativas em saúde: contribuições teóricas. Cad. Saúde Publica. 2008; 24(1):17-27.

18. Slade GD. Derivation andvalidation of a short-form oralhealth impact profile. CommunityDent Oral Epidemiol. 1997; 25: 284-90.

19. World Health Organization.Oral health surveys: basic methods. 5th ed: WHO; 2013. Disponível em: www.who.int/about/licensing/copyright_form/en/index.html. Acesso em:[06/06/2017].

20. Campos ACV, Vargas AMD, Ferreira EF. Satisfação com saúde bucal de idosos brasileiros: um estudo de gênero com modelo hierárquico. Cad. Saúde Pública. 2014; 30(4): 757-72.

21. Lefèvre F, Lefèvre AMC. O discurso do sujeito coletivo: um novo enfoque em pesquisa qualitativa (desdobramentos). Caxias do Sul: EDUCS; 2003.

22. Lefèvre F, Lefèvre AMC. O sujeito coletivo que fala.Interface Comum. Saúde Educ. 2006; 10(20):1-8.

23. Lefèvre F, Lefèvre AMC. Depoimentos e discursos:uma proposta de análise em pesquisa social. Brasília: Editora Liber; 2005.

24. Andrade KLC, Ferreira EF. Avaliação da inserção da odontologia no Programa Saúde da Família de Pompéu (MG): a satisfação do usuário. Ciênc. Saúde Coletiva. 2006;11(1):123-130.

25. Pinto RS, Matos DL, Loyola Filho AI. Característicasassociadas ao uso de serviços odontológicos públicospela população adulta brasileira. Ciênc. Saúde Coletiva. 2012; 17(2): 53244.

26. Costa EBC. Satisfação dos usuários assistidos em Centros de Especialidades Odontológicas Regionais do Ceará, Brasil. [Dissertação]. Campinas (SP): Faculdade São Leopoldo Mandic; 2016. 
27. Fonseca LLV, Nehmy RMQ, Mota JAC. O valor social dos dentes e o acesso aos serviços odontológicos. Ciênc. Saúde Coletiva. 2015; 20(10): 3129-38.

28. Cascaes AM. Doença periodontal e a autoavaliação da saúde bucal em adultos brasileiros. [Dissertação]. Florianópolis (SC): Universidade Federal de Santa Catarina; 2008.

29. Brasil. Ministério da Saúde. Secretaria de Atenção à Saúde. Secretaria de Vigilância em Saúde. SB Brasil 2010: Pesquisa Nacional de Saúde Bucal: resultados principais. Brasília: Ministério da Saúde; 2012.

30. Unfer B, Saliba O. Avaliação do conhecimento popular e práticas cotidianas em saúde bucal. Rev. Saúde Pública. 2000; 34: 190-5.

31. Costa EB, Carneiro VSM, Pedrosa HLO, Godoy GP, Amorim JA. Dor de dente: percepção dos usuários da atenção básica de saúde. Odontologia Clín. Científ. 2009; 8(1): 53-58.

32. Celich KLS, Spadari G. Estilo de vida e saúde: condicionantes de um envelhecimento saudável. Cogitare Enferm. 2008; 13(2): 252-60.

Artigo apresentado em outubro de 2017 Artigo aprovado em julho de 2019 Artigo publicado em julho de 2020 